SCHWERPUNKT

\section{Technik und Pflege in einer Gesellschaft des langen Lebens}

\section{Einführung in den Schwerpunkt}

von Manfred Hülsken-Giesler, Philosophisch-Theologische Hochschule Vallendar, und Bettina-Johanna Krings, Institut für Technikfolgenabschätzung und Systemanalyse (ITAS), Karlsruhe

Reflexionen zum Technikeinsatz in Kontexten der Pflegearbeit in einer Gesellschaft des langen Lebens lassen sich nicht auf pragmatische Aspekte der Technikentwicklung, -nutzung und -bewertung begrenzen. Sie fordern vielmehr konstitutiv zu einer Auseinandersetzung mit grundlegenden Fragen der gesellschaftlichen Entwicklung auf und provozieren Debatten um den gesellschaftlichen Umgang mit Alter und Vulnerabilität oder um das Verhältnis von Effektivität und Effizienz der Fürsorge in einer modernen Gesellschaft. Wie durch ein Brennglas bündeln sich Fragen nach den kulturellen Vorstellungen des Alterns sowie der gesellschaftlichen Entwicklung insgesamt in der Auseinandersetzung um Pflege und Technik in einer Gesellschaft des langen Lebens. Das vorliegende Schwerpunktheft sucht Anregungen zu diesen Fragen aus Perspektive der Gerontologie, der Pflegewissenschaft sowie der Technikfolgenabschätzung zu erhalten.

Reflections on the use of technologies in the context of care in a society of longer living cannot be limited to pragmatic aspects of technology development, use or assessment. Instead these issues demand a constitutive discussion over the basic questions of society's development and provoke debates on the societal way of dealing with age and vulnerability as well as the relationship between effectively and efficiency and care in a modern society. Like under a magnifying glass, questions of the cultural notions of age or societal development as a whole arise through the discourses surrounding care and technology in a society of longer living. The following thematic focus searches for approaches to these questions from perspectives of gerontology, nursing sciences as well as technology assessment.

\section{Technik in einer Kultur des langen Lebens}

Der Titel des vorliegenden Themenschwerpunktes „Technik und Pflege in einer Gesellschaft des langen Lebens" ist bewusst gewählt und trägt zwei Botschaften. Zum einen knüpft der Titel an öffentliche Debatten und wissenschaftliche Diskurse an, die sich mit technischen Innovationen in Pflegekontexten beschäftigen und angesichts der Diagnose einer „Überalterung“ von Gesellschaften in nahezu allen hochindustrialisierten Gesellschaften eine hohe Aktualität besitzen. Die diskursive Verknüpfung von Technik und Pflege weist hierbei auf vielseitige Erwartungen, die angekündigten Versorgungsprobleme in der Pflege technisch (auf)zulösen. Diese Erwartungen werden auf der nationalen genauso wie auf der internationalen Ebene formuliert und prägen die Ausrichtung von politischen Forschungs- und Innovationsstrategien (EC 2007) in besonderem Maße. Zum anderen zielt der Titel auf die Anforderungen des demografischen Alterns per se, deren „historisch präzedenzloser Zugewinn an durchschnittlicher Lebenszeit" (Kocka et al. 2009, S. 9) im Laufe der letzten Jahrzehnte darauf hinweisen, dass sich das Lebensalter der Menschen in hochindustrialisierten Gesellschaften signifikant verlängert hat. Ohne hier näher auf die Gründe einzugehen, werden so „Gesellschaften des langen Lebens" (vgl. auch Kocka et al. 2009) generiert, die die Sozialstruktur von Gesellschaften sowie deren institutionalisierte Lebensphasen in Bildungs-, Erwerbs- und Ruhestandsphasen (Kohli 1985; Kohli 2009) signifikant verändert haben und weiterhin verändern werden. Diese Veränderungen haben einschneidende Folgen auf die Generationenverhältnisse, welche familiäre und zivilgesellschaftliche Strukturen genauso berühren wie institutionelle Rahmenbedingungen im Hinblick auf die Gestaltung von Betreuung, Begleitung, Versorgung und Pflege.

Die „Verwirklichungen des Alters" (Schroeter 2014) als Vorstellung und Wirklichkeit sind 
hierbei vielfältig und auf mindestens zwei Ebenen angesiedelt: auf der Ebene der Innenperspektive als subjektive Wahrnehmung eines jeden Menschen auf den eigenen Alterungsprozess als leiblicher, emotionaler und geistiger Transformationsprozess (vgl. in der Prosa prominent Beauvoir 2014). Diese Ebene wird ergänzt durch die Ebene der Außenperspektive (Buchen/Maier 2008; Amann/Kolland 2014), die individuelle, zivilgesellschaftliche und institutionelle Aspekte als soziokulturelle Deutungsmuster des Alterns in den Blick nimmt. Die Komplexität der Innenperspektive spiegelt hierbei folgerichtig die Komplexität der Außenperspektive auf diese Prozesse wider, in der ständig neue Vorstellungen über das Altern erzeugt werden. Diese Vorstellungen entsprechen freilich den jeweiligen soziokulturellen Rahmenbedingungen von Gesellschaften und können im internationalen Vergleich sehr unterschiedlich ausfallen. Wird beispielsweise „eine neue Doxa eines aktiven, erfolgreichen oder produktiven Alterns auf die sozialpolitische Agenda gehoben" (Schroeter 2014, S. 282), so verlängert sich diese auch in die Einschätzung darüber, was mit Blick auf individuelle Beispiele als ,gelungenes Alter“ oder „erfolgreiches Alter" interpretiert werden kann (Künemund 2009). Umgekehrt werden Ansprüche für Sicherung und Pflege formuliert, die in vielen gesellschaftlichen Krisendiagnosen derzeit zu alarmistischen Einschätzungen zukünftiger Versorgungsengpässe und somit zu eher bedrohlichen Vorstellungen des Alters führen.

Diese Einschätzungen gelten in hohem Maße für die Phase des Alters, in denen Menschen auf Pflege und Fürsorge ihrer Mitmenschen angewiesen sind. Unabhängig davon, ob diese Fürsorge im familiären oder institutionellen Rahmen stattfindet, wird besonders in diesen Pflegekontexten eine „Kultur des Alters“ (Schroeter 2014, S. 282; Kohli 2009) konstruiert, die kulturelle und soziale Bedeutungsgehalte dieser Lebensphase generiert und in kollektive Bilder über das Altern und in konkrete Handlungsstrategien übersetzt. Die eingangs dargestellte Erwartung, technologische Artefakte in diese Pflegekontexte verstärkt einzubinden, eröffnet vor diesem Hintergrund neue Sichtweisen darüber, wie und vor welchen normativen Bewertungen diese Lebensphase in den Gesamtverlauf des Lebens eingebettet wird.
So verhandeln Diskurse zur Technisierung der Pflege immer auch grundsätzliche Fragen darüber, welchen Stellenwert die Fürsorge im gesellschaftlichen Gesamtgefüge einnimmt. Wie und vor welchen Zielen werden Technologien in Pflegekontexte eingesetzt? Welche pflegerischen Handlungen sollen technisiert, also umgestaltet oder auch ersetzt werden? Wer wird mit der Erwartung der Technisierung der Pflege adressiert? Wie wird der Einsatz von Technologien in Pflegekontexten genutzt, um die (letzten) Phasen des Lebens zu bereichern? Wer entscheidet und wie begründet sich die Frage, was in diesem Kontext eine „Bereicherung“ darstellt?

Vor diesen Fragen und Überlegungen knüpfen die Autoren ihren Themenschwerpunkt „Technik und Pflege in einer Gesellschaft des langen Lebens" an die These, dass die Erwartungen über die Technisierung von Pflegekontexten nicht losgelöst von wissenschaftlich und öffentlich geführten Diskursen über kulturelle Vorstellungen des Alterns sowie der gesellschaftlichen Entwicklung insgesamt verhandelt werden können. Die Frage der Gestaltung von Pflegekontexten ist damit nicht vorrangig in Perspektive von Expertenkulturen in verschiedenen Teilsystemen der Gesellschaft zu verhandeln, sie fordert vielmehr die gesamte Gesellschaft dazu heraus, grundlegende Wertvorstellung etwa mit Blick auf die Bedeutung von Gemeinschaft, Leistungsvermögen oder Alter zu reflektieren und neu zu diskutieren. Die Frage der Gestaltung von Pflegekontexten unter Bedingungen des Technikeinsatzes ist an Fragen der Qualität des langen Lebens anzuknüpfen. Angesichts des ungebrochenen demografischen Alters scheint also nicht nur Kreativität im Hinblick auf neue Technologien gefragt. Die Entwicklungen berühren zunehmend (auch) die Neugestaltung des Zusammenwirkens der Generationen, was in besonderem Maße die Neugestaltung der sozialen Sicherungssysteme moderner Gesellschaften nach sich zieht (vgl. Kocka et al. 2009). Diesbezüglich werden technische Innovationen derzeit eher konservativ in bestehende bzw. in Erprobung befindliche sozialrechtliche Regelungen eingedacht (vgl. Bundesministerium für Gesundheit 2013). Um das Potenzial computergestützter Technologien ,als wichtige Bausteine zur Bewältigung der demografischen Herausfor- 
derungen in einer modernisierten Gesellschaft" (Hülsken-Giesler/Bleses 2015, S. 3) zu entfalten, wären sie dagegen offensiver im Zusammenhang mit der Re-Organisation von Pflege als gesamtgesellschaftliche Aufgabe zu denken.

\section{Pflege von (alten) Menschen als technische Herausforderung?}

Die überwiegend negativ konnotierten Prognosen einer sog. „Überalterung von Gesellschaften“ weisen im Rahmen der Debatten um die Technisierung der Pflege auf zukünftige Pflegenotstände, die - spiegelt man die demografische Entwicklung an heutigen Pflegekapazitäten - zunächst nicht von der Hand zu weisen sind. Geht man von diesen statistischen Korrelationen aus, so wird beispielsweise für Deutschland angenommen, dass schon in zwei Jahrzehnten eine größere Anzahl älterer Menschen im Verhältnis zu jüngeren Menschen leben und langfristig versorgt werden müssen. So weisen eine Reihe statistischer Hochrechnungen darauf hin, dass im Jahre 2050 die Zahl der Pflegebedürftigen zwischen 3,3 und 4,4 Mio. Menschen anzusiedeln ist, während die Zahl heute bei etwa 2,6 Mio. pflegebedürftiger Menschen liegt (Statistisches Bundesamt 2009; Blinkert/Gräf 2009). Diese Zahlen weisen vor dieser quantitativen Lesart darauf hin, dass ein gesteigerter Bedarf an Pflege für Menschen im letzten Lebensalter notwendig sein wird, der sich - wiederum gespiegelt an der derzeitigen Pflegesituation - als problematisch gestalten könnte (vgl. kritisch Krings et al. 2014). Diese Prognosen entfalten ihre alarmierende Wirkung jedoch in einer Logik, „die nur Demografie als sich wandelnd annimmt und alles andere als statisch" (Krings et al. 2014, S. 75). Mögliche migrationspolitische, arbeitsmarkt- oder sozialpolitische Strukturveränderungen, die auf die Gesamtentwicklung einwirken (könnten), bleiben hier weitgehend unberücksichtigt (Saraceno 2009; Ammann/Kolland 2014).

Darüber hinaus scheint es auch sinnvoll zu sein, die gesamtgesellschaftliche Pflegesituation von alten Menschen vor einer qualitativen Lesart zu betrachten und den Blick verstärkt darauf zu lenken, welche Probleme im Rahmen dieser Pflegekontexte auftreten. Hierbei können eine Vielzahl von „Pflegearrangements“ (Blinkert/
Klie 2004) identifiziert werden, d. h. soziale Netzwerke, die die Pflege abdecken. Es zeigt sich schnell, dass sich gerade in der Pflege alter Menschen der Anteil häuslicher, ambulanter und stationärer Versorgung stark durchmischt. Tatsächlich werden aktuell zwei Drittel des Gesamtbedarfs an Pflege im häuslichen Bereich, also durch Kinder und Lebenspartnerinnen bzw. Lebenspartner erbracht. Dieses Modell wird nicht nur von den Betroffenen selbst, sondern auch von der Politik aus Kostengründen präferiert. So kann als Prognose für die zukünftige Pflege v. a. festgehalten werden, dass die Richtlinie ,häusliche vor stationärer Pflege“ (Blinkert/Gräf 2009) weiterhin groBen Bestand haben wird. Vor diesem Hintergrund zielen politische und innovationsstrategische Vorgehensweisen vermehrt auf den Bereich der ambulanten Pflege, um alten Menschen zu ermöglichen, so lange wie möglich in den gewohnten vier Wänden zu bleiben und ,selbstbestimmt zu leben" (Fraunhofer 2014; Krings 2014). Prominente Ansätze sind hier etwa Ambient Assisted Living-Systeme (AAL), also technische Systeme, die sich „situationsspezifisch den Bedürfnissen des Benutzers" (ebda., S. 44) anpassen sollen. Darüber hinaus werden auf der Basis avancierter technischer Vernetzungsmöglichkeiten zunehmend mehr „Optionen“ (ebda., S. 44) in Betreuungssystemen ausgelotet. Vor allem im Bereich technisch basierter Monitoring-, Planungs- und Kontrollsysteme sowie im Bereich technischinteraktiver Systeme werden Potenziale für das pflegerische Handeln vermehrt ausgelotet (Althammer/Sehlbach 2012; Weinberger et al. 2014) und auch für die Zukunft in Aussicht gestellt.

Im Bereich der Pflege existieren Erfahrungen im Hinblick auf elektronisch gestützte Informationssysteme seit ca. 30 Jahren v. a. im Bereich der akutstationären Versorgung (Hülsken-Giesler 2008; Hielscher et al. 2014). Vor der Erwartung, die Arbeitsproduktivität zu erhöhen sowie die Arbeitsabläufe in der Pflege ,effizienter“ zu gestalten, hat sich hier die computergestützte Dokumentation in der Praxis der Pflegearbeit weitgehend durchgesetzt. ${ }^{1}$ So fußen „Krankenhausinformationssysteme [fußen] auf der Generierung und Bereitstellung von Daten für die Unterstützung des Krankenhausmanagements sowie von klinischen Daten für die medizinische Leistungser- 
bringung" (Hielscher et al. 2014, S. 10). Hierbei ist die Einführung systematischer Pflegemodelle und Prozessbeschreibungen der Pflegepraxis inzwischen Teil der komplexen Anforderungen, die von den Prüfbehörden detailliert nachgefragt werden (MDS 2005). Die bisherigen Erfahrungen mit technisch basierter Unterstützung dieser Arbeitsprozesse werden vor einer pflegewissenschaftlichen Perspektive unterschiedlich bewertet und vor unterschiedlichen Fragestellungen (auch) kontrovers diskutiert. Was diese Bewertungen zeigen, ist, dass der Einsatz von (neuen) Technologien und ihre Auswirkungen die Arbeitskontexte verändern und erwünschte und unerwünschte Effekte gleichermaßen bewirken (Manzei 2009). „Es sind also über die Dokumentationssysteme nicht nur die konkrete Leistung und die Aktivitäten der Pflegearbeit zu erfassen, sondern ebenso soll die Qualität der Pflegepraxis über fein differenzierte Abfragen sichergestellt werden." (Hielscher et al. 2014, S. 9ff., s. auch Ammenwerth et al. 2002) Gerade die Technisierung der Arbeitsprozesse im Pflegekontext deutet darauf hin, dass Ansprüche an ein effizientes Pflegemanagement in vielerlei Hinsicht kontraproduktiv zu den Erfordernissen der Pflege sind. Effizientes Pflegemanagement argumentiert und berechnet Ressourcen auf der Basis rational begründeter und eindeutig operationalisierter Daten und Kennzahlen. Arbeitswissenschaftliche wie pflegewissenschaftliche Erkenntnisse verweisen jedoch darauf, dass berufliches Pflegehandeln als subjektivierendes Arbeitshandeln $\mathrm{zu}$ verstehen ist, das neben planbaren und rational begründbaren Aspekten konstitutiv durch situatives und exploratives Vorgehen charakterisiert ist und neben distanzierend kognitiv-rationalen Begründungen auch komplexe sinnliche - also körperlich-leibliche - Wahrnehmungen in die berufliche Entscheidungsfindung einbezieht. Vorrationale, alltagsweltlich begründete Wissensformen erhalten ebenso wie Kommunikation, Beziehungs- und Gefühlsarbeit eine konstitutive Bedeutung für die Pflegearbeit in lebenspraktischen Bezügen und können daher keineswegs einseitig instrumentalisiert oder als Residualkategorien gegen rationale Begründung des Pflegehandelns ausgespielt und aus dem Wissenskanon des professionellen Handelns ausgegrenzt werden (vgl. ausführlich
Hülsken-Giesler 2008; Böhle/Weishaupt 2003; vgl. auch Remmers in diesem Heft).

Obwohl computergestützte Systeme aufgrund ihrer binären Logik eben diese konstitutiven Bestandteile der Pflegearbeit bisher nur unzureichend abzubilden in der Lage sind, haben sich hochspezialisierte technische Systeme zur Pflegedokumentation v. a. in jüngster Zeit in Krankenhäusern weitgehend durchgesetzt. Dies ist in der stationären und ambulanten Altenpflege bislang nicht der Fall, hier wird noch „ein gewisser ,time-lag' vermutet [werden], so dass hier der Prozess der Umstellung auf digitale Dokumentation mit all seinen Vor- und Nachteilen noch in vollem Gange ist" (Hielscher et al. 2014, S. 10).

Ein großes Feld im Rahmen der Technisierungsdebatten deckt der Themenbereich „Pflege von Menschen mit Demenz" ab, da aufgrund des demografischen Alterns hier ein signifikanter Anstieg von pflegebedürftigen Menschen in den nächsten Jahren zu erwarten ist (Alzheimer's Disease International 2013). Zum anderen führt der Verlust der kognitiven und kommunikativen Fähigkeiten der Menschen mit Demenz zum Ausschluss aus dem gesellschaftlichen Leben, was v. a. auf Seiten der betreuenden Personen - Angehörigen und Pflegepersonal - zu extrem hohen Belastungen führt (Beer et al. 2014). Hier sind in den letzten Jahren eine Fülle von interdisziplinären Pilotprojekten durchgeführt worden, die auf der Basis von Navigationssystemen, robotischen Assistenzsystemen (z. B. Robbe Paro) oder Teleüberwachungssystemen vielfältige Optionen im Rahmen der Betreuung von Menschen mit Demenz entwickeln und/oder bewerten (Böhle/ Pfadenhauer 2014). „Vor allem im Rahmen von Unterstützungsleistungen, Kommunikations- und Überwachungsaufgaben, aber teilweise ebenso zu sozial-interaktiven, logistischen oder kommunikativen Zwecken" (Ziegler et al. 2015, S. 38) soll langfristig das Potenzial von technischen Optionen vermehrt erschlossen werden.

Als zentrale Frage erscheint hierbei, inwiefern diese technischen Optionen im Rahmen einer emotionsorientierten und empathischen Pflege und Betreuung von Menschen mit Demenz in eine sinnvolle Anwendung gebracht werden können (ebda., S. 38). Obgleich es hier vielfach positive Einschätzungen über das Potenzial anbe- 
raumter Technologien in diesen Pflegekontexten gibt, bleibt eine Vielzahl ethischer Anforderungen bestehen, die die „Einbindung von vulnerablen Personen“" (ebda., S. 48) in Forschungsprojekte, aber auch in Technikentwicklungsprozesse stark berühren. Hier gilt es, diese Anforderungen in interdisziplinären Forschungszusammenhängen sichtbar zu machen, um die Möglichkeiten, aber auch die Grenzen technischer Optionen im Feld der (Alten-)Pflege aufzuzeigen.

\section{Pflege und Fürsorge von (alten) Menschen als gesellschaftliche Herausforderung}

Nicht nur in der Literatur, sondern auch im Rahmen der hier versammelten Beiträge gibt es Konsens darüber, dass Technologien grundsätzlich wichtige Elemente in der Ausgestaltung der Pflegekontexte sowohl in der stationären, als auch in der ambulanten Pflege sind: Sie werden zukünftig eine systematische Bedeutung im Pflegemix von informellen und professionell Pflegenden sowie im Zusammenspiel der Mikro-, Meso- und Makroebene des Pflegewesens erhalten. Während die akutstationäre Pflege schon seit Jahrzehnten Technisierungsschübe durchläuft und deren Pflegepraxis weitgehend in technischen Umgebungen stattfindet, wird in der langzeitstationären sowie der ambulanten Pflege aus Sicht der Forschung und Entwicklung ein großer Nachholbedarf für die Einführung von computergestützten Technologien in die verschiedenen Pflegekontexte erkannt. Gleichzeitig dient die Förderung von Technikentwicklungsprogrammen auch explizit dem Ziel der Erschließung von neuen Märkten im Pflegesektor.

Diese Bestrebungen werden von forschungspolitischer Seite flankiert und vor dem Hintergrund ausgerichtet, die technologischen Innovationen an einer ,bedarfsgerechten“ Pflege auszurichten. Die Vorstellung, neue Technologien an den Bedürfnissen und Bedarfen der unterschiedlichen Akteure der Pflegearrangements auszurichten, scheint ebenfalls weitgehend Konsens zu sein. Unterzieht man diese Diskurse jedoch einer genaueren Betrachtung, so fällt in vielen Fällen auf, dass ,...the deeper philosophical questions concerning the meaning of the end of life experience are passed over in favour of concentrating on achieving technical solutions to problems defined in terms amenable to such solutions" (Sparrow/ Sparrow 2006, S. 156, s. auch Krings et al. 2014).

Besonders vor dem Hintergrund, dass sich die Gesundheitsversorgung zunehmend als „technisch-wissenschaftlich-ökonomisch überformte gestaltet" (Hülsken-Giesler 2008, S. 26), passen sich die oben dargestellten Erwartungen an eine Technisierung der Pflege hier nahtlos ein. Dennoch plädieren die Autoren dafür, im Themenfeld „Technik und Pflege“ einen Perspektivenwechsel zu vollziehen und diesen Diskurs in gesamtgesellschaftliche Problemstellungen von Gesellschaften des langen Lebens einzubinden. So kann das Handlungsfeld „Technik und Pflege" in einer Kultur des Alters um institutionelle, arbeitsmarktpolitische und psychologische Aspekte etc. bereichert werden, was völlig neue Handlungsräume - auch für technologische Entwicklungen - eröffnet. Vor diesem Hintergrund setzten sich auch die Beiträge des vorliegenden Schwerpunktes aus unterschiedlichen Disziplinen (Gerontologie, Pflegewissenschaft, Technikfolgenabschätzung) zusammen, um den Blick für das Themenfeld sowie für relevante, zukünftige Fragestellungen im Themenfeld zu öffnen.

\section{Die einzelnen Beiträge}

Hartmut Remmers diskutiert grundlegende Herausforderungen, aber auch Begrenzungen des $\mathrm{Zu}$ sammenspiels von Pflege und Technik unter philosophisch-anthropologischen Gesichtspunkten und zieht Schlüsse aus einer pflegewissenschaftlichen Perspektive. Pflege als existenzielle, elementare Tätigkeit der individuellen und gesellschaftlichen Reproduktion ist demnach konstitutiv auf technische Unterstützung verwiesen, hat aber ihren Eigensinn gegen Verselbständigungstendenzen moderner Technologien zu verteidigen, um nicht der technisch-instrumentellen Logik von Institution, Bürokratie und Ökonomie zu verfallen.

Andreas Kruse und Eric Schmitt verdeutlichen in gerontologischer Perspektive die Möglichkeiten moderner Technologien zur Begründung und Realisierung einer altersfreundlichen Kultur, die insbesondere Aspekte der selbständigen Lebensführung, aber auch die besonderen Bedarfe von Menschen mit demenziellen Erkran- 
kungen berücksichtigt. Potenziale der technologischen Entwicklung werden dabei sowohl für diagnostisch-therapeutische Kontexte einer professionellen Pflege und Versorgung wie auch für Zusammenhänge einer auf Teilhabe und Lebensqualität fokussierten Pflegearbeit identifiziert.

In gerontologischer Perspektive befürwortet Harald Künemund durchaus die vielseitigen technischen Möglichkeiten, die die Lebensqualität von alten Menschen verbessern helfen (könnten). Darüber hinaus seien Technologien auch hilfreich, um präventive Maßnahmen in den Bereichen ,Rehabilitation“" und „Pflege“ zu unterstützen. Allerdings, und dies zeigten die Erfahrungen mit dem Einsatz von Technik, gäbe es große Probleme in der faktischen Umsetzung. Insgesamt gerieten im Rahmen von wissenschaftlichen Bedarfserhebungen nicht die Bedürfnisse der alten Menschen in den Blick, was zu einer fehlenden Problemevaluation, einer fehlenden Technikakzeptanz und einer fehlenden Prozessevaluation führe. Die Bearbeitung dieser Defizite könnte, nach seiner Ansicht, die Versorgungsstrukturen von alten Menschen insgesamt deutlich verbessern.

Nora Weinberger und Michael Decker argumentieren in der Perspektive der Technikfolgenabschätzung aus einer ähnlichen Richtung wie Harald Künemund. Sie konstatieren, dass es im Rahmen der aktuellen Diskussionen um eine Technisierung der (Alten-)Pflege hauptsächlich darum gehen sollte, das „gap“ zwischen technologischen Entwicklungen und den sog. Bedarfen der unterschiedlichen Anwenderinnen und Anwender in Pflegesituationen zu schließen. Sie schlagen in diesem Kontext das Konzept der ,bedarfsorientierten" Technikentwicklung vor, das dezidiert technische Lösungen in Abstimmung mit den dort vorgefundenen sozialen Bedarfslagen erheben (soll). Am Beispiel der stationären Pflege von Menschen mit Demenz wird dieser Ansatz exemplifiziert.

Mit einem umfassenden Einblick in die „Zukunft der Pflege“" stellen Manfred HülskenGiesler sowie Bernd Wiemann Ergebnisse aus einem Projekt vor, das mit der empirischen Methode der Szenarienentwicklung zukünftige Modelle gesellschaftlicher Versorgungsstrukturen im Jahr 2053 ermittelt. Auffallend ist hier, wie sehr soziale Werte und Vorstellungen von "Pflege“ und „Alter“ die verschiedenen Szenarien prägen. Die rhetorische Zuspitzung der einzelnen Szenarien macht hierbei deutlich, wie diese kulturellen Werte die Zukunftsgestaltung beeinflussen und wie dezidiert öffentliche Debatten vonnöten sind, um gesellschaftlich die ,Zukunft von Gesellschaften des langen Lebens" vorzubereiten.

\section{Anmerkung}

1) Anvisiert wird damit insbesondere der Anschluss von pflegerelevanten Daten an einrichtungsspezifische und gesundheitssystemische Kommunikationen, um übergreifende Steuerungsprozesse auch in der Pflege zu ermöglichen. Voraussetzung dieser Entwicklung ist allerdings, dass das Wissen der Pflege in Form von standardisierten und codierbaren Fachsprachen an die technische Logik computergestützter Kommunikationen angeschlossen wird. Im Kontext der Langzeitpflege steht diese Entwicklung noch weitgehend aus. Kritisch wird diskutiert, dass die Transformation von pflegerelevanten Wissensbeständen in computerkompatible Fachsprachen relevante Wissensformen der Pflege (Erfahrungswissen, implizites Wissen etc.) systematisch ausblendet und das pflegerische Handeln damit einseitig auf ein rational begründetes Problemlösungshandeln verpflichtet (vgl. Hülsken-Giesler 2008).

\section{Literatur}

Althammer, T.; Sehlbach, O., 2012: Mehr schlecht als Recht. Zum aktuellen Stand von Datenschutz und Datensicherheit in der Pflege und im Sozialwesen 2012. Ergebnisse einer Befragung von 295 Leitungskräften in stationären Einrichtungen in Deutschland. Burgwedel

Alzheimer's Disease International, 2013: World Alzheimer Report 2013. Journey of Caring. An Analysis of Long-term Care for Dementia; http://www. alz.co.uk/research/WorldAlzheimerReport2013.pdf (download 24.7.15)

Amann, A.; Kolland, F. (Hg.), 2014: Das erzwungene Paradies des Alters? Weitere Fragen an eine Kritische Gerontologie. Wiesbaden

Ammenwerth, E.; Iller, C.; Mahler, C. et al., 2002: Einflussfaktoren auf die Akzeptanz und Adoption eines Pflegedokumentationssystems. PIK-Studie 2002 - Universitätsklinikum Heidelberg. Innsbruck

Beauvoir, S. de, 2014: Das Alter. Reinbek

Beer, Th.; Bleses, H.; Ziegler, S., 2014: Personen mit Demenz und robotische Assistenzsysteme. Ethnografische Erkundungen zu Randakteuren der Pflege. In: 
Hülsken-Giesler, M.; Bleses, H.M. (Hg.): Schwerpunkt: Technologisierung der Pflegearbeit? Wissenschaftlicher Diskurs und Praxisentwicklungen in der stationären und ambulanten Pflege. In: Pflege \& Gesellschaft 20/1 (2015), S. 20-36

Blinkert, B.; Gräf, O., 2009: Deutsche Pflegeversicherungen vor massiven Herausforderungen. Deutsche Bank Research; http://wwwdbreseach.dePROD/DBR INTERNET DE-PROD/ PROD0000000000239350.pdf (download 22.7.15)

Blinkert, B.; Klie, T., 2004: Solidarität in Gefahr: Pflegebereitschaft und Pflegebedarfsentwicklung im demografischen und sozialen Wandel. Hannover

Böhle F.; Weishaupt S., 2003: Unwägbarkeiten als Normalität - die Bewältigung nichtstandardisierbarer Anforderungen in der Pflege durch subjektivierendes Handeln. In: Büssing, A.; Glaser, J. (Hg.): Dienstleistungsqualität und Qualität des Arbeitslebens im Krankenhaus. Göttingen, S. 149-162

Böhle, K.; Pfadenhauer, M. (Hg.), 2014: Editorial. Social Robots Call for Social Sciences. In: Science, Technology \& Innovation Studies 10/1 (2014), S. 3-10 Buchen, S.; Maier, M.S. (Hg.), 2008: Älterwerden neu denken. Interdisziplinäre Perspektiven auf den demografischen Wandel. Wiesbaden

Bundesministerium für Gesundheit, 2013: Unterstützung Pflegebedürftiger durch technische Assistenzsysteme. Abschlussbericht. Berlin

EC - European Commission, 2007: Action Plan on Information and Communication Technologies and Ageing. Brüssel

Fraunhofer-Magazin ,weiter.vorn “, 2014: Zeitschrift für Forschung, Technik und Innovation 2 (2014). München

Hielscher, V.; Kirchen-Peters, S.; Sowinski, Ch., 2015: Technologisierung der Pflegearbeit? Wissenschaftlicher Diskurs und Praxisentwicklung in der stationären und ambulanten Langzeitpflege. In: Pflege \& Gesellschaft 20/1 (2015), S. 5-19

Hülsken-Giesler, M., 2008: Der Zugang zum Anderen. Zur theoretischen Rekonstruktion von Professionalisierungsstrategien pflegerischen Handelns im Spannungsfeld von Mimesis und Maschinenlogik. Göttingen

Hülsken-Giesler, M.; Bleses, H.M., 2015: Neue Technologien in der Pflege - Einleitung in den Schwerpunkt. In: Pflege \& Gesellschaft 20/1 (2015), S. 3-4

Kocka, J.; Kohli, M.; Streeck, W., 2009: Einführung, In: Kocka, J.; Kohli, M.; Streeck, W. (Hg.): Altern: Familie, Zivilgesellschaft, Politik. Akademiegruppe Altern in Deutschland. Stuttgart, S. 9-12
Kohli, M., 1985: Die Institutionalisierung des Lebenslaufs. Historische Befunde und theoretische Argumente. In: Kölner Zeitschrift für Soziologie und Sozialpsychologie 37/1 (1985), S. 1-29

Kohli, M., 2009: Familiale Generationenbeziehungen im Wohlfahrtsstaat. In: Kocka, J.; Kohli, M.; Streeck, W. (Hg.): Altern: Familie, Zivilgesellschaft, Politik. Akademiegruppe Altern in Deutschland. Stuttgart, S. 91-113

Krings, B.-J., 2014: Technische Assistenz- und Pflegesysteme in Zeiten des demografischen Wandels. Ein Beitrag aus sozialwissenschaftlicher Perspektive. In: Technikfolgenabschätzung - Theorie und Praxis 23/2 (2014), S. 81-87

Krings, B.-J.; Böhle, K.; Decker, M. et al., 2014: Kurzstudie Serviceroboter in Pflegearrangements. Demografischer Wandel und die Zukunft der Pflegearrangements. In: Decker, M.; Fleischer, T.; Schippl, J. et al. (Hg.): Zukünftige Themen der Innovationsund Technikanalyse. Lessons Learned und ausgewählte Ergebnisse. Karlsruhe, S. 63-122

Künemund, H., 2009: Erwerbsarbeit, Familie und Engagement in Deutschland. In: Kocka, J.; Kohli, M.; Streeck, W. (Hg.): Altern: Familie, Zivilgesellschaft, Politik. Akademiegruppe Altern in Deutschland. Stuttgart, S. 19-39

Manzei, A., 2009: Neue betriebswirtschaftliche Steuerungsformen im Krankenhaus. Wie durch die Digitalisierung der Medizin ökonomische Sachzwänge in der Pflegepraxis entstehen. In: Pflege \& Gesellschaft 14/1 (2009), S. 38-53

MDS-Medizinischer Dienst der Spitzenverbände der Krankenkassen e.V., 2005: Grundsatzstellungnahme: Pflegeprozess und Dokumentation. Essen

Saraceno, Ch., 2009: The Impact of Ageing on Intergenerational Family Relationship in the Context of Different Family and Welfare Regimes. In: Kocka, J.; Kohli, M.; Streeck, W. (Hg.): Altern: Familie, Zivilgesellschaft, Politik. Akademiegruppe Altern in Deutschland. Stuttgart, S. 115-132

Schroeter, K.R., 2014: Verwirklichungen des Alters? In: Amann, A.; Kolland, F. (Hg.): Das erzwungene Paradies des Alters? Weitere Fragen an eine Kritische Gerontologie. Wiesbaden, S. 283-318

Sparrow, R.; Sparrow, L., 2006: In the Hands of Machines? The Future of Aged Care. In: Mind and Machines 16/2 (2006), S. 141-161

Statistisches Bundesamt, 2009: Bevölkerung Deutschlands bis 2060. 12. Koordinierte Bevölkerungsberechnung. Wiesbaden

Weinberger, N.; Decker, M.; Krings, B.-J., 2014: Pflege von Menschen mit Demenz - Bedarfsorientierte 
Technikgestaltung. In: Schultz, T.; Putze, F.; Kruse, A. (Hg.): Technische Unterstützung für Menschen mit Demenz. Karlsruhe, S. 61-74

Ziegler, S.; Treffurth, T.; Bleses, H.M., 2015: Entsprechend dem (mutmaßlichen) Willen? Ethische Anforderungen bei der Einbindung von vulnerablen Personen (am Beispiel von Personen mit Demenz) in wissenschaftlichen Projekten zur Beforschung emotionsorientierter Pflege und Betreuung mit robotischen Assistenzsystemen. In: Hülsken-Giesler, M.; Bleses, H.M. (Hg.): Schwerpunkt: Technologisierung der Pflegearbeit? Wissenschaftlicher Diskurs und Praxisentwicklungen in der stationären und ambulanten Pflege. In: Pflege \& Gesellschaft 20/1 (2015), S. 37-52

\section{Kontakt}

Prof. Dr. Manfred Hülsken-Giesler

Pflegewissenschaftliche Fakultät

Philosophisch-Theologische Hochschule Vallendar

Pallottistraße 3, 56179 Vallendar

E-Mail: mhuelsken-giesler@pthv.de

Dr. Bettina-Johanna Krings

Institut für Technikfolgenabschätzung und Systemanalyse (ITAS)

Karlsruher Institut für Technologie (KIT)

Karlstraße 11, 76133 Karlsruhe

Tel.: +49 721 608-26347

E-Mail: bettina-johanna.krings@kit.edu

$《 \gg$

\section{Natürlichkeit und Künstlichkeit \\ Zur Analyse und Bewertung von Technik in der Pflege des Menschen}

von Hartmut Remmers, Fachbereich Humanwissenschaften, Universität Osnabrück

In diesem Beitrag werden Möglichkeiten und Grenzen des Technikeinsatzes in der Pflege unter mehreren philosophisch-anthropologischen Prämissen aufgezeigt: Pflegerische Tätigkeiten sind elementare Formen der Reproduktion des Lebens und durch strukturelle Eigensinnigkeit (z. B. zyklischer Modus) gekennzeichnet. Sie gestalten sich allerdings unter Bedingungen historisch geschaffener und variierender Lebensformen mit Unterstützung technischer Instrumente. Den künstlich erzeugten Systemen des Lebens (Institutionen) wohnt ebenso wie der Technik eine Tendenz der Verselbständigung inne. Illustrieren lässt sich das an der Geschichte der Pflege, welche durch Trends einer wachsenden technischen Umgestaltung gekennzeichnet ist. Hinter diesen Trends verbergen sich indessen Kollisionen zweier unvereinbarer Eigenlogiken: einer Logik persönlicher Zuwendung und Hilfe, die nur wenig formalisierbar ist, und einer gegenläufigen Logik von Ökonomie sowie planender Verwaltung und Organisation. Nicht zufällig verweisen diese Inkommensurabilitäten auf Grenzen bspw. des Einsatzes moderner Informations- und Kommunikationstechnologien in der Pflege hilfebedürftiger Menschen, die durch ethische und rechtliche Garantien wie Schutz der Persönlichkeit oder körperliche Integrität markiert sind.

In this contribution prospects and constraints of the use of technology in care are pointed out under various philosophical and anthropological premises. Care activities are fundamental forms of the reproduction of life and are characterised by structural obstinacy (e.g. cyclic mode). However, they emerge under conditions that are historically created and due to the assistance of technical instruments are constantly varying. The tendency of self-perpetuation is inherent to both artificially generated systems of life (institutions) as well as technology. The history of care, characterised by tendencies of a growing technical transformation, 